\title{
Contact heat evoked potentials using simultaneous EEG and fMRI and their correlation with evoked pain Katherine Roberts ${ }^{1}$, Anastasia Papadaki ${ }^{1}$, Carla Gonçalves ${ }^{2}$, Mary Tighe ${ }^{3}$, Duncan Atherton ${ }^{1}$, Ravikiran Shenoy ${ }^{1}$, Donald McRobbie ${ }^{1}$ and Praveen Anand*1
}

\author{
Address: ${ }^{1}$ Imperial College Healthcare NHS Trust, London, UK, ${ }^{2}$ East Kent Hospitals NHS Trust, Canterbury, UK and ${ }^{3}$ Kings College London, \\ London, UK \\ Email: Katherine Roberts - k.roberts05@imperial.ac.uk; Anastasia Papadaki - a.papadaki@imperial.ac.uk; \\ Carla Gonçalves - carla.sag@gmail.com; Mary Tighe - mary@brainvision.co.uk; Duncan Atherton - duncanatherton@hotmail.co.uk; \\ Ravikiran Shenoy - r.shenoy@imperial.ac.uk; Donald McRobbie - d.mcrobbie@imperial.ac.uk; Praveen Anand* - p.anand@imperial.ac.uk \\ * Corresponding author
}

Published: 17 December 2008

BMC Anesthesiology 2008, 8:8 doi:10.1 186/|47|-2253-8-8

This article is available from: http://www.biomedcentral.com/I47|-2253/8/8

(c) 2008 Roberts et al; licensee BioMed Central Ltd.

This is an Open Access article distributed under the terms of the Creative Commons Attribution License (http://creativecommons.org/licenses/by/2.0), which permits unrestricted use, distribution, and reproduction in any medium, provided the original work is properly cited.
Received: 7 February 2008

Accepted: 17 December 2008

\begin{abstract}
Background: The Contact Heat Evoked Potential Stimulator (CHEPS) utilises rapidly delivered heat pulses with adjustable peak temperatures to stimulate the differential warm/heat thresholds of receptors expressed by $A \delta$ and $C$ fibres. The resulting evoked potentials can be recorded and measured, providing a useful clinical tool for the study of thermal and nociceptive pathways. Concurrent recording of contact heat evoked potentials using electroencephalogram (EEG) and functional magnetic resonance imaging (fMRI) has not previously been reported with CHEPS. Developing simultaneous EEG and fMRI with CHEPS is highly desirable, as it provides an opportunity to exploit the high temporal resolution of EEG and the high spatial resolution of fMRI to study the reaction of the human brain to thermal and nociceptive stimuli.
\end{abstract}

Methods: In this study we have recorded evoked potentials stimulated by $51{ }^{\circ} \mathrm{C}$ contact heat pulses from CHEPS using EEG, under normal conditions (baseline), and during continuous and simultaneous acquisition of fMRI images in ten healthy volunteers, during two sessions. The pain evoked by CHEPS was recorded on a Visual Analogue Scale (VAS).

Results: Analysis of EEG data revealed that the latencies and amplitudes of evoked potentials recorded during continuous fMRI did not differ significantly from baseline recordings. fMRI results were consistent with previous thermal pain studies, and showed Blood Oxygen Level Dependent (BOLD) changes in the insula, post-central gyrus, supplementary motor area (SMA), middle cingulate cortex and pre-central gyrus. There was a significant positive correlation between the evoked potential amplitude (EEG) and the psychophysical perception of pain on the VAS.

Conclusion: The results of this study demonstrate the feasibility of recording contact heat evoked potentials with EEG during continuous and simultaneous fMRI. The combined use of the two methods can lead to identification of distinct patterns of brain activity indicative of pain and pronociceptive sensitisation in healthy subjects and chronic pain patients. Further studies are required for the technique to progress as a useful tool in clinical trials of novel analgesics. 


\section{Background}

Functional magnetic resonance imaging (fMRI) has developed into a tool that is extensively used in non-invasive brain imaging. It provides information about cerebro-vascular activity throughout the whole brain with excellent spatial localisation, yet it is limited by the poor temporal resolution it offers, which is in the order of seconds. On the other hand, electroencephalogram (EEG) is recorded directly from the scalp of the subject and can provide information about neurophysiological activity with a very precise temporal resolution, in the order of milliseconds. The disadvantage of EEG however, is that localisation of the source of electrical activity within the brain is quite difficult.

Therefore the integration of these two techniques is highly desirable, and would allow the exploitation of the advantages of both techniques - high spatial resolution of fMRI and high temporal resolution of EEG. The technique would be widely applicable in all areas of neurophysiological research, but particularly in pain studies, where combined use of the two techniques could lead to the identification of distinct patterns of brain activity indicative of pain and pro-nociceptive sensitisation in healthy subjects and chronic pain patients. These patterns could prove useful in the assessment of the analgesic efficacy of novel analgesic compounds, adding to the desirability of co-registration of pain evoked potentials (such as those stimulated by contact heat) with EEG and fMRI.

Objective markers of pain could be less variable and/or more sensitive to analgesic treatments (i.e. EPs, fMRI). The present study enables the use of these markers to simultaneously assess pharmacodynamic-pharmacokinetic relationships and antihyperalgesic activity in single dose studies in experimental pain models in humans, and in pain patients (e.g. of novel agents that block TRPV1, the heat and capsaicin receptor). Pain biomarkers are needed to provide early pivotal information on efficacy, doseresponse and time-course of TRPV1 antagonists, for strategic rapid and cost-effective drug development. Further, neuropathic pain patients would be expected to have reduced pain-evoked potential amplitudes but increased fMRI activation, different from volunteer or inflammatory models/conditions.

The use of simultaneous EEG and fMRI in pharmacological studies of novel analgesics would allow (i) measurement of the combined response to the same pharmacological intervention when the plasma concentration of the analgesic is at its peak (i.e. at the same timecourse); (ii) recordings can be analysed at the single subject level, reducing inter-session variability, which is well recognised with regard to pain scores, and (iii) selection of the parameter which enables possible reduction in dose in clinical trials.

Simultaneous EEG and fMRI has already been utilised in studies of epilepsy [1], sleep [2], studies of human alpha activity $[3,4]$ and the study of auditory $[5,6]$, visual $[7,8]$, and motor activity [9]. In the field of pain research, laser and electrically stimulated pain evoked potentials have been successfully co-registered using EEG and fMRI $[10,11]$, but CHEPS has the advantages of a larger area of stimulation, the ability to apply repetitive stimuli to the same cutaneous area without inducing erythema, and simplicity of use in the clinic (i.e. no eye protection required, easy to move location); however, one disadvantage of CHEPS is that in order to avoid habituation the thermode must be physically moved between stimuli.

Simultaneous EEG/fMRI is a readily feasible application yet the MR environment poses a number of technical challenges, limitations and safety issues (for a review of technical and safety issues see $[12,13])$. The gradient switching fields and radiofrequency (RF) pulses of the MR scanner can create currents in conducting loops that can potentially cause heating of the electrodes and burns at the point of contact with the scalp of the subject. Therefore it is recommended to avoid loops and crossing over of electrode wires, and introduce current limiting resistors to electrode wires.

The gradient fields and RF pulses of the MR scanner can lead to artefacts that can obscure the signal recorded from the EEG, as can movements within the static magnetic field; movement of the head, talking, swallowing and even the pulsatile motion of the heart (ballistocardiogram artefact). The magnet's helium cold pump and gradient coils also produce mechanical vibrations that can be picked up by the EEG wires in the static magnetic field and converted to electrical noise. All these issues need to be addressed and overcome during experimental set-up and processing of EEG and fMRI data, in terms of patient safety and also preserving the quality of the data collected.

Until recently, quantifiable contact heat evoked potentials in EEG had been hard to elicit, due to technical limitations i.e. slow temperature rise and fall times. However, the contact heat evoked potential stimulator (CHEPS) has been designed with a maximum (adjustable) temperature rise time of $70^{\circ} \mathrm{C} / \mathrm{s}$. CHEPS can stimulate the differential thermal thresholds of receptors innervated by A $\delta$ and $\mathrm{C}$ nociceptive nerve fibres, and has been shown to selectively excite these fibre subtypes in human hairy and glabrous skin [14]. The latencies and amplitudes of heat evoked A $\delta$ potentials stimulated by CHEPS have been shown to be robust and reproducible $[15,16]$, despite the disadvantage posed by the slow temperature rise time of 
the heat stimuli produced by CHEPS $\left(70^{\circ} \mathrm{C} / \mathrm{s}\right.$ compared to a rise time of greater than $1000^{\circ} \mathrm{C} / \mathrm{s}$ reported with lasers) which may lead to a reduction in temporal summation and thus a less synchronous afferent volley [17].

The compatibility of CHEPS with fMRI further widens its scope of application both in healthy volunteers for research purposes and in chronic pain patients. Reproducibility of blood oxygen level dependent (BOLD) responses and pain scores to CHEPS stimulation have already been illustrated in healthy volunteers [18].

In this human volunteer study we have assessed the feasibility of monitoring contact heat evoked A $\delta$ potentials with simultaneous EEG and fMRI, and determined their relationship to the ratings of evoked pain.

\section{Methods \\ Subjects}

Ten healthy volunteers were recruited to take part in this feasibility study ( 6 female, 4 male). The average age of participants was 27.7 years (range 22 - 35 years). Informed consent was taken from all subjects prior to commencement of the study and the study itself was approved by Hammersmith and Queen Charlotte's \& Chelsea Research Ethics Committee (REC reference 06/ Q0404/9).

\section{Heat stimuli}

Contact heat stimulation was performed using CHEPS (Medoc Ltd, Ramat Yishai, Israel) with a thermode area of $572.5 \mathrm{~mm}^{2}$, and a heating thermo-foil (Minco Products, Inc., Minneapolis, $\mathrm{MN}$ ) covered with a $25 \mu \mathrm{m}$ layer of thermo conductive plastic (Kapton ${ }^{\circledast}$, thermal conductivity at $23^{\circ} \mathrm{C}$ of $\left.0.1-0.35 \mathrm{~W} / \mathrm{m} / \mathrm{K}\right)$. The thermode heating rate was up to $70^{\circ} \mathrm{C} / \mathrm{s}$, the cooling rate up to $40^{\circ} \mathrm{C} / \mathrm{s}$ and the baseline temperature was $32^{\circ} \mathrm{C}$.

\section{Experimental setup}

\section{Baseline protocol}

Baseline recording of evoked potentials was undertaken with the subject lying on the scanner table (outside the magnet) without any scanning taking place. Ten $51^{\circ} \mathrm{C}$ stimuli of approximately $800 \mathrm{~ms}$ duration (approximately $271 \mathrm{~ms}$ to reach peak temperature of $51^{\circ} \mathrm{C}$ and approximately $475 \mathrm{~ms}$ to return to baseline [total $746 \mathrm{~ms}$ ]) and 7 $\mathrm{s}$ inter-stimulus interval were applied to the left volar forearm of the subject, and the thermode moved after each stimulus to avoid habituation.

\section{fMRI protocol}

An event-related protocol was used during continuous fMRI acquisition. The protocol consisted of thirty-two $51^{\circ} \mathrm{C}$ stimuli of approximately $800 \mathrm{~ms}$ duration, where the inter-stimulus interval varied between 8 and $32 \mathrm{~s}$. Stimuli were again applied to the left volar forearm, and the thermode moved after each stimulus (Figure 1). A well recognized adverse feature of CHEPS is that placement of the thermode on the skin can cause concomitant activation of mechanoreceptors in the skin. Movement of the thermode after each heat pulse was deemed necessary in order to prevent habituation of the subjects to the stimulus [19], however as this is not synchronised with the paradigm we do not anticipate that it will result in any BOLD response.

Baseline and fMRI protocols were repeated on two occasions on all subjects to assess reproducibility.

\section{Pain ratings}

After completion of baseline and fMRI protocols, subjects were asked to rate the pain of contact heat stimulation by CHEPS on a visual analogue scale (VAS), by placing a mark on a line $10 \mathrm{~cm}$ in length that was graded from 0 to 10,0 being not painful at all, and 10 being the worst pain

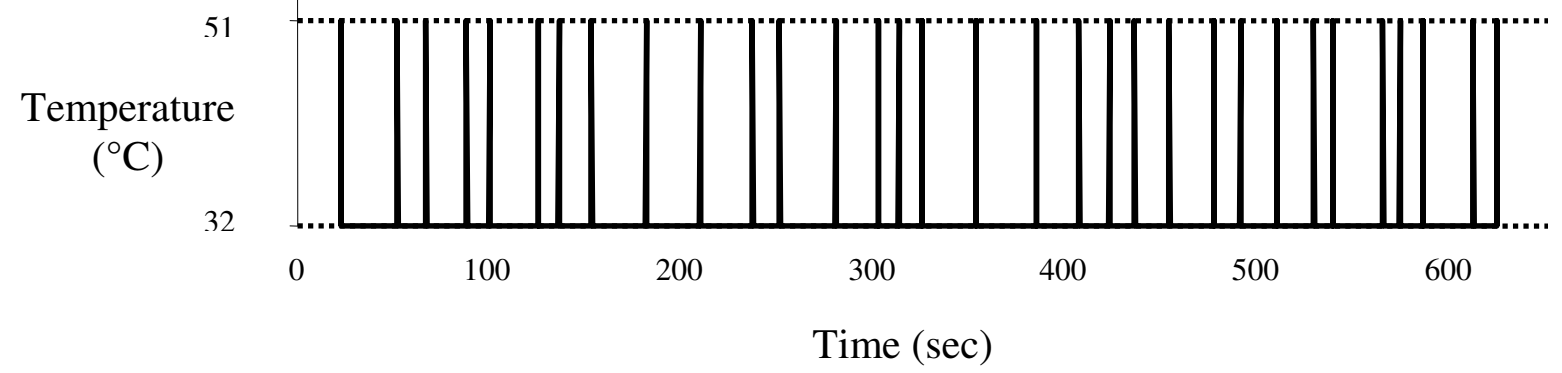

Figure I

Event related paradigm. The protocol during continuous fMRI acquisition consisted of thirty-two $5 \mathrm{I}^{\circ} \mathrm{C}$ stimuli (from a baseline of $32^{\circ} \mathrm{C}$ ) of approximately $800 \mathrm{~ms}$ duration. The inter-stimulus interval varied between 8 and $32 \mathrm{~s}$. 
imaginable. The distance of the mark from 0 (in $\mathrm{cm}$ ) was measured and noted as the pain score.

\section{Electroencephalogram (EEG) recording}

EEG was acquired from a 32 electrode cap using MRI compatible equipment (BrainCap MR, BrainAmp MR Plus 32 Amplifier, BrainProducts GmbH, Munich, Germany) with the subject relaxed and eyes opened. Electrodes were contacted with Abralyt 2000 electrode gel and impedance maintained below $5 \mathrm{k} \Omega$ (when the value of the $5 \mathrm{k} \Omega$ current limiting safety resistors [in place as standard on each electrode lead, close to the electrode itself] was subtracted). The data was acquired in a mono-polar fashion, which avoids the disadvantages of average reference recordings and allows for re-montaging of data after acquisition. The reference electrode was located in the $\mathrm{FCz}$ position, and the ground electrode in the Iz position. Vertical electro-oculogram (EOG) and electro-cardiogram (ECG) were monitored to allow exclusion of traces contaminated by eye blinks and to enable pulse artefact subtraction. EEG signals were digitised and transmitted to acquisition equipment outside the scanner room by optical fibre. For the baseline recording (outside the scanner) a sampling rate of $250 \mathrm{~Hz}$ was applied, and for fMRI recording (inside the scanner) a sampling rate of $5000 \mathrm{~Hz}$ was applied. Online low and high pass filters (10 s and $250 \mathrm{~Hz}$ respectively) were also applied to the EEG data recorded during baseline and fMRI protocols. CHEPS stimulation and scan events were registered on a trigger channel connected to the acquisition equipment.

EEG data was analysed using a dedicated software package (BrainVision Analyser Version 1.05.0002, BrainProducts GmbH, Munich, Germany). The baseline EEG data was filtered (low pass $0.5305 \mathrm{~Hz}$, high pass $40 \mathrm{~Hz}$ ), segmented around the trigger input from CHEPS, corrected for blinks using the Gratton and Coles correction algorithm, averaged (10 segments), and re-referenced to an average reference. A baseline correction was applied from -200 to $0 \mathrm{~ms}$ pre-stimulus.

The EEG data recorded during fMRI was corrected for scan artefacts using an MRI correction solution provided with the analysis software, using subtraction algorithms based on methods originally developed by Allen and colleagues [20]. Scan start points were detected and marked (using average gradient and criterion for continuous scans) and corrected (using template drift correction). Pulse artefacts were also removed using a solution provided with the analysis software (correction by $\mathrm{R}$ peaks - demeaned amplitude algorithm used for $\mathrm{R}$ peak detection). Independent component analysis (ICA) was carried out on all data sets, and trials contaminated by subject movement or ocular artefacts were identified visually and removed in the ICA back transform. The data was segmented, aver- aged (33 segments), re-referenced and a baseline correction applied (as above for the baseline recorded data).

All EEG data is reported from the $\mathrm{Cz}$ electrode, with an average reference. The latency of heat evoked A $\delta$ potentials was measured from the first definitive negative peak (N2) and the amplitude measured peak to peak - N2 to P2 (the N2 to P2 component was defined as the peak within a time window of $250-550 \mathrm{~ms}$ ).

Graphs were created and statistical tests performed on EEG data using GraphPad Prism (Version 3.02 for Windows, GraphPad Software, San Diego California USA).

\section{fMRI acquisition}

In each scanning session, 275 fMRI scans were acquired on a 1.5 T Siemens Avanto magnetic resonance scanner (Siemens Medical Systems, Erlangen, Germany) with a standard head array coil. 19 slices parallel to the anterior commisure and the posterior commisure (AC/PC line) were acquired using a gradient echo EPI (Echo Planar Imaging) sequence (Repetition time/Echo time $(\mathrm{TR} / \mathrm{TE})=$ $2.3 \mathrm{~s} / 53 \mathrm{~ms}$, flip angle $=90^{\circ}$, field-of-view $(\mathrm{FOV})=23 \mathrm{~cm}$, matrix $=64 \times 64$, voxel size $=3.6 \mathrm{~mm} \times 3.6 \mathrm{~mm} \times 5 \mathrm{~mm})$. A high resolution T1-MP-RAGE anatomical scan was also obtained $\left(\mathrm{TR} / \mathrm{TE}=11 \mathrm{~ms} / 5.2 \mathrm{~ms}\right.$, flip angle $=15^{\circ}, \mathrm{FOV}=$ $23 \mathrm{~cm}$, matrix $=256 \times 256$, voxel size $=0.9 \mathrm{~mm} \times 0.9 \mathrm{~mm}$ $\times 1 \mathrm{~mm})$.

To achieve synchronization, the trigger output of the scanner was used to initialize the fMRI paradigm and triggers from CHEPS stimulation and the scanner were recorded together with the EEG signals.

\section{fMRI processing}

Processing of fMRI images was performed using SPM5 (Functional Imaging Laboratory, London, UK). Due to T1 saturation effects, the first 5 volumes of each acquisition were discarded, leaving 270 volumes. Each dataset was realigned to correct for any motion during the acquisition, time corrected due to differences in image acquisition time between slices, followed by normalisation to transform the data to match the SPM template. The template images supplied with SPM conform to the space defined by the ICBM, NIH P-20 project, and approximate that of the space described in the atlas of [21]. Finally, images were smoothed using an isotropic Gaussian Kernel $(8 \mathrm{~mm}$ full-width-at-half-maximum).

Statistical analysis was based on the General Linear Model (GLM). For within subject analysis the scanning paradigm was specified in SPM and a first level model estimation was performed. The event responses were modelled onto a design matrix by specifying their onset times and their duration. For the group analysis of the ten subjects, a 
canonical, random effects model (RFX) was used to make a population inference (corrected $\mathrm{p}<0.05$ ). Spatial extend thresholding (voxel threshold $=135 \mathrm{~mm}^{3}$ ) was carried out to exclude isolated voxels or small groups and only show clusters of activation. An average brain, representative of all 10 subjects was determined by averaging the normalised structural brain volumes. Significantly activated regions resulting from the group analysis were superimposed on the average brain. The location of the activated regions was assessed using SPM Anatomy toolbox.

To assess correlation between fMRI activation and evoked potential amplitude (or VAS scores), values collected for each subject were entered as covariates using simple regression analysis in SPM5.

\section{Results \\ Contact heat evoked potentials}

Evoked potentials were successfully recorded using EEG in all subjects during baseline recordings and nine subjects during fMRI acquisition (the data of one subject could not be used due to technical problems). The waveform recorded was similar in both protocols (Figure 2a, b).

The average evoked potential latencies for the first baseline protocol were (mean \pm SEM) N2: $0.316 \pm 0.009 \mathrm{~s}$, P2: $0.429 \pm 0.015 \mathrm{~s}$, and for the second baseline protocol repeated on a separate occasion were N2: $0.322 \pm 0.010 \mathrm{~s}$, P2: $0.432 \pm 0.013 \mathrm{~s}$. The latencies for the first fMRI protocol were N2: $0.314 \pm 0.008 \mathrm{~s}, \mathrm{P} 20.446 \pm 0.017 \mathrm{~s}$, and for the second, N2: $0.317 \pm 0.009 \mathrm{~s}, \mathrm{P} 2: 0.443 \pm 0.015 \mathrm{~s}$. These latencies were approximately $0.100 \mathrm{~s}$ longer than those reported in LEP studies, and this is most likely due to the slower temperature rise time of CHEPS (and thus longer stimulus duration) in comparison to laser stimuli, which may lead to slower activation of nociceptors and a reduction of temporal summation $[17,22]$.

The average amplitudes for baseline protocols 1 and 2 were $24.01 \pm 2.95 \mu \mathrm{V}$ and $25.82 \pm 3.30 \mu \mathrm{V}$ respectively. The amplitudes for fMRI protocols were $24.87 \pm 3.73 \mu \mathrm{V}$ for fMRI 1 and $24.14 \pm 4.26 \mu \mathrm{V}$ for fMRI 2. Comparisons of evoked potential latencies and amplitudes revealed no significant differences between the two baseline and fMRI sessions (Figure 3 ).

Comparisons of pooled baseline and fMRI protocol data did not reveal any difference between latencies and amplitudes (Table 1). There was also no difference in the baseline to peak amplitudes of the baseline and fMRI data (N2 baseline vs. fMRI $\mathrm{p}=0.3748$; $\mathrm{P} 2$ baseline vs. fMRI $\mathrm{p}=$ 0.4456).

Differences in overall averages for evoked responses were noted between males and females, with evoked potentials recorded from females having a faster latency (F $0.302 \pm$ $0.004 \mathrm{~s}$ vs. $\mathrm{M} 0.345 \pm 0.009 \mathrm{~s}, \mathrm{p}=0.0003)$ and a larger amplitude (F $27.62 \pm 2.87 \mu \mathrm{V}$ vs. $\mathrm{M} 18.47 \pm 1.78 \mu \mathrm{V}, \mathrm{p}=$ $0.0286)$.

No significant correlation between subject age and overall evoked potential latency or amplitude was observed $\left(\mathrm{r}_{\mathrm{s}}=\right.$ $0.2298, \mathrm{p}=0.2567 ; \mathrm{r}_{\mathrm{s}}=0.1043, \mathrm{p}=0.3925$ respectively).

\section{fMRI}

Group analysis of fMRI data for all ten subjects, collected continuously throughout the fMRI protocol and CHEPS recording revealed areas of Blood Oxygen Level Dependent (BOLD) activation upon CHEPS stimulation bilaterally in the insula, post-central gyrus, and SMA, and contralateral to the site of stimulation (i.e. right) in the middle cingulate cortex and pre-central gyrus (Figure 4, Table 2).

FMRI responses to CHEPS heat stimulation are reproducible across multiple sessions and this is demonstrated in Figure 5 showing group results of each session

Although the thermode was moved randomly, it is possible that there could be some contribution of the touch sensation to the measured BOLD response. To avoid this, the movement of the thermode should be modelled as a nuisance covariate in future experiments.

\section{Pain ratings}

Stimulation at $51^{\circ} \mathrm{C}$ with the CHEPS probe elicited a painful sensation in all ten subjects. There was no significant difference between reported VAS ratings for baseline and fMRI protocols (Table 1).

Female subjects were noted to have a significantly higher VAS than males for CHEPS stimulation $(\mathrm{p}=0.0416)$. No correlation between age and average reported VAS was observed $\left(r_{s}=0.1534, p=0.3410\right)$.

\section{Correlations}

Correlation of evoked potential amplitude with VAS (for all data, baseline and fMRI protocols repeated twice) revealed a significant, positive relationship, $\mathrm{r}_{\mathrm{s}}=0.5956, \mathrm{p}$ $<0.0001$ (Figure 6a). A significant, positive correlation was also seen when the baseline and fMRI data was pooled to give an overall evoked potential amplitude and VAS score for each of the ten subjects, $r_{s}=0.7697, p=$ 0.0063 (Figure 6b).

The regression analysis in SPM showed no correlation between BOLD signal and evoked potential amplitudes or VAS scores. 


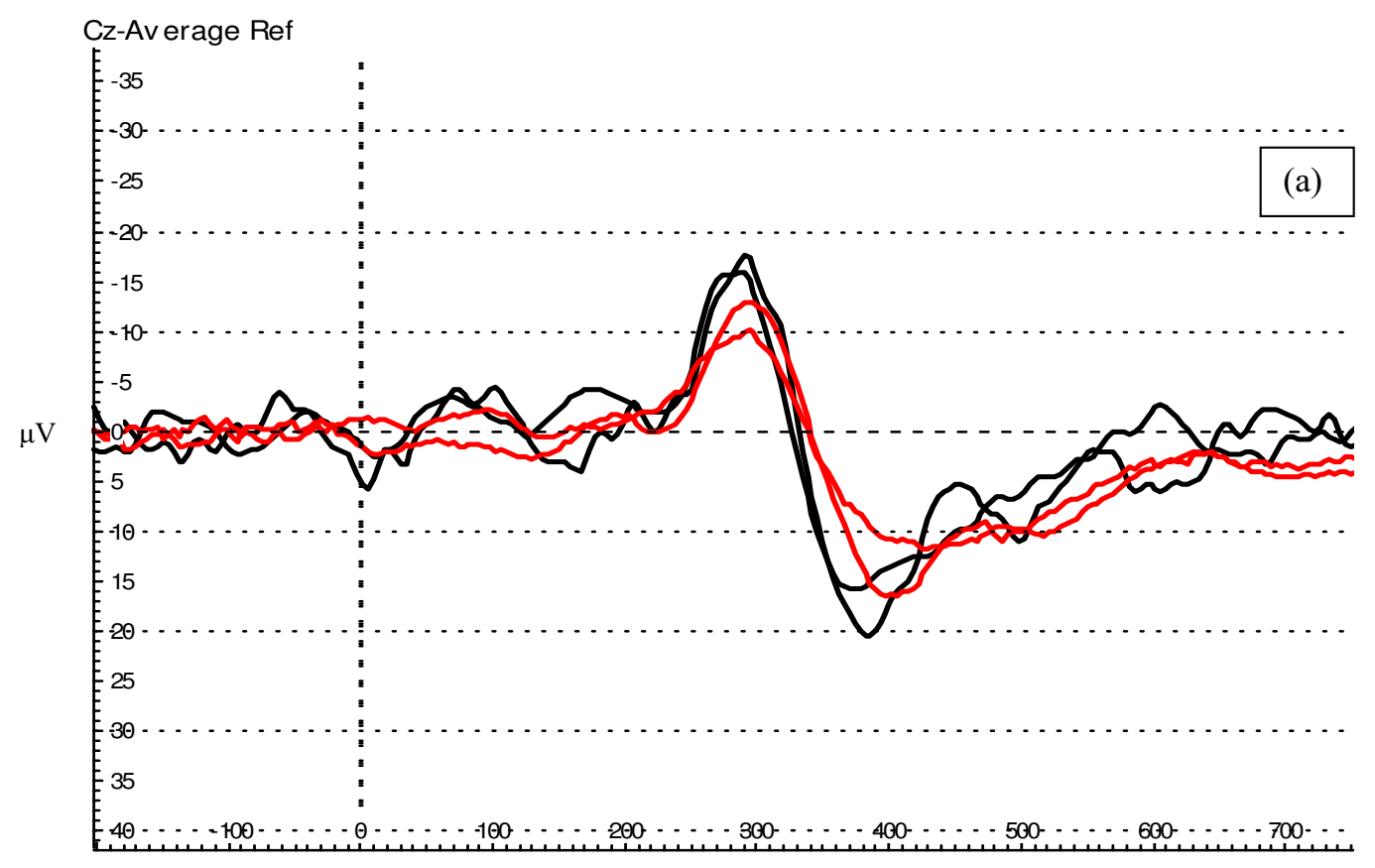

$\mathrm{ms}$

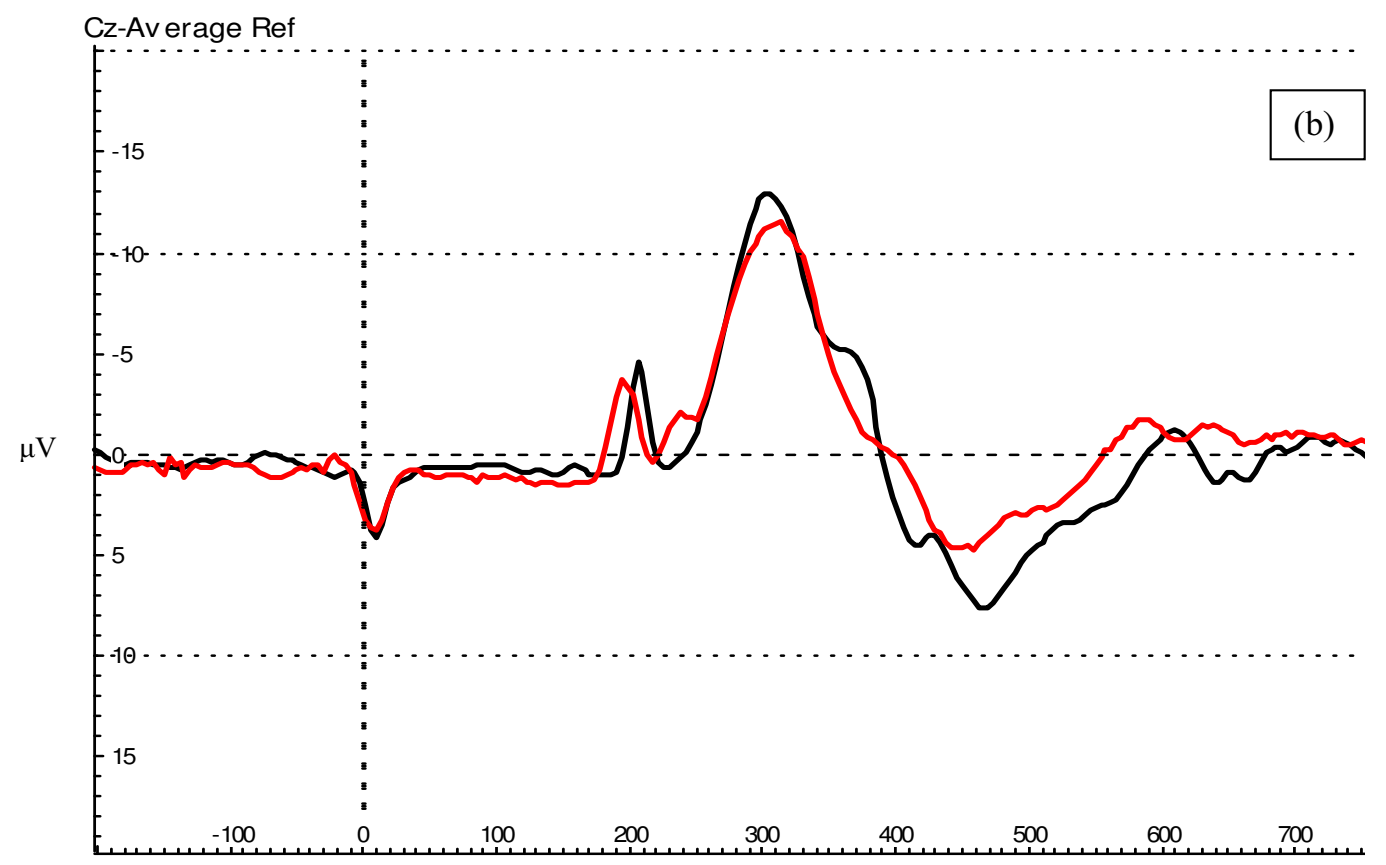

$\mathrm{ms}$

\section{Figure 2}

Evoked potential waveforms. (a) Evoked potentials recorded during baseline (black traces) and fMRI (red traces) protocols in subject \# 5. (b) Evoked potentials (grand average for all subjects) recorded at baseline (I plus 2) (black trace) and during fMRI (I plus 2) protocols (red trace). The deflections on the trace occurring at 0 and $250 \mathrm{~ms}$ are artefacts produced by the stimulator. 

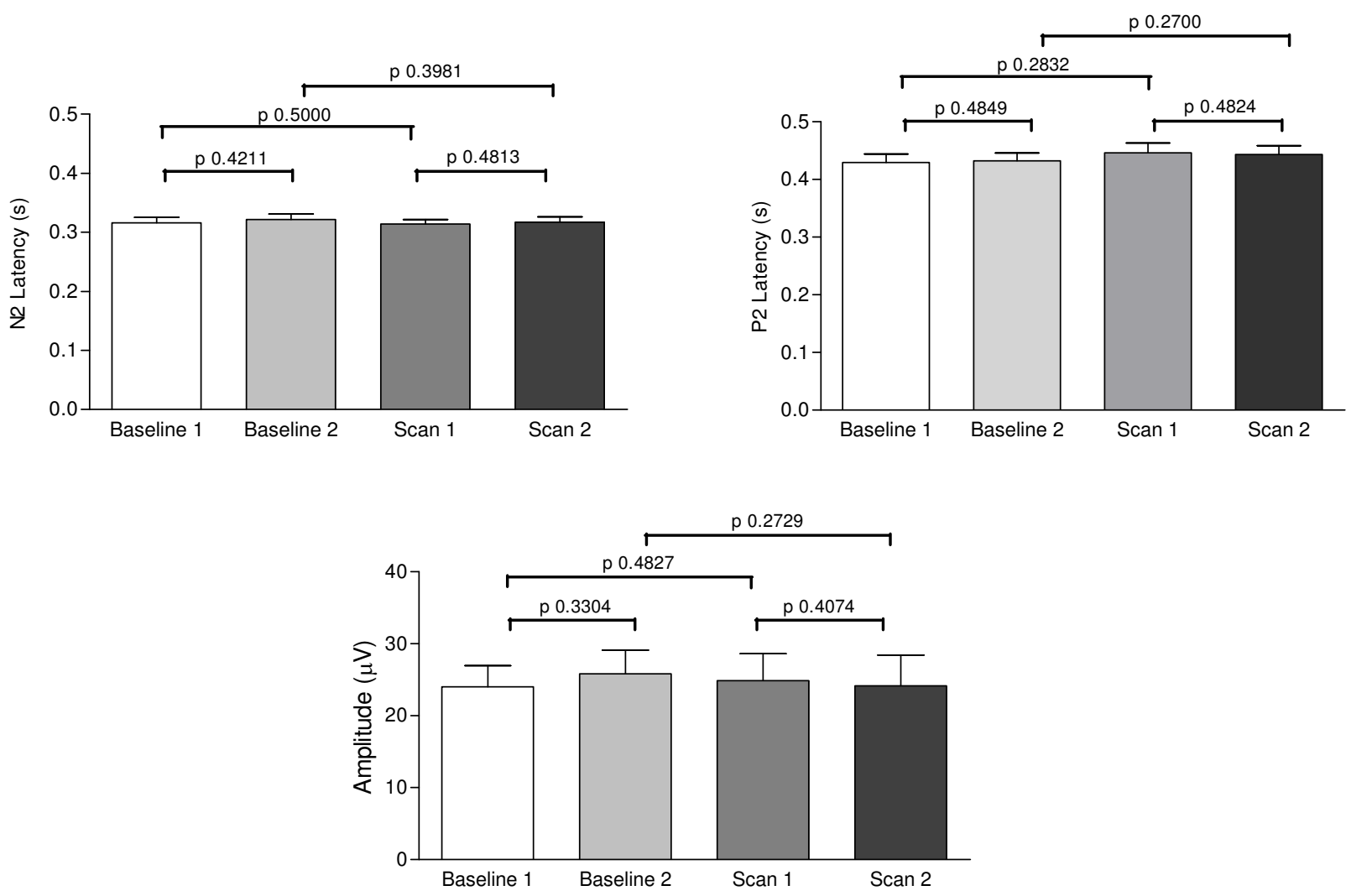

Figure 3

Latency and amplitude of evoked potentials in baseline and fMRI protocols. Graphical representation of the N2 and P2 latencies $(s)$ and amplitudes $(\mu \mathrm{V})$ recorded in the two separate baseline and $\mathrm{fMRI}$ sessions. There were no differences in either parameter when recorded under baseline conditions or during continuous $\mathrm{fMRI}$.

\section{Discussion}

In this study we have demonstrated that it is feasible to record brain activity in response to noxious thermal stimulation $\left(51^{\circ} \mathrm{C}\right.$ contact heat) with CHEPS, using simultaneous EEG and fMRI. The EEG recorded during fMRI acquisition was unaffected by the additional processing steps required to remove MRI related artefacts. These arte- facts can contaminate the raw data and their removal can compromise the quality of the EEG signal. However, our simultaneously acquired EEG revealed heat evoked A $\delta$ potentials that were reproducible across the two separate fMRI sessions. Although the aim of this feasibility study was not to directly compare the measurements inside and outside the scanner, the amplitudes and latencies

Table I: Evoked potentials and pain scores recorded during baseline and fMRI protocols in healthy volunteers.

\begin{tabular}{llll}
\hline Protocol & N2 latency $(\mathbf{s})$ & Amplitude $(\mu \mathbf{V})$ & VAS (0-10) \\
\hline Baseline & $0.319 \pm 0.006$ & $24.87 \pm 2.15$ & $5.30 \pm 0.53$ \\
\hline FMRI & $0.317 \pm 0.006$ & $24.49 \pm 2.77$ & $5.97 \pm 0.57$ \\
\hline Difference & $n s(p=0.4183)$ & $n s(p=0.3402)$ & ns $(p=0.1918)$ \\
\hline
\end{tabular}

Mean \pm SEM

ns $=$ not significant

Average data $(n=10)$ for evoked potential latency, amplitude and pain scores during baseline and fMRI protocols. There were no significant differences between the results obtained in the baseline and fMRI sessions. 
Table 2: Areas of BOLD fMRI activation after stimulation using CHEPS.

\begin{tabular}{llllll}
\hline Cluster & $\mathbf{T}$ & $\mathbf{X}$ & $\mathbf{z}$ & Anatomical regions \\
\hline Cluster I (50I vox) & & & & & \\
\hline 1 & 9.82 & 0 & -10 & 65 & Left SMA \\
\hline 2 & 8.66 & 24 & -16 & 74 & Right Superior Frontal Gyrus \\
\hline 3 & 7.69 & 30 & -25 & 68 & Right Precentral Gyrus \\
\hline 4 & 7.46 & 15 & -13 & 68 & Right SMA \\
\hline 5 & 7.43 & 12 & -13 & 53 & Right Middle Cingulate Cortex
\end{tabular}

\section{Cluster 2 (366 vox)}

\begin{tabular}{llllll}
\hline 1 & 9.55 & 54 & -28 & 38 & Right SupraMarginal Gyrus \\
\hline 2 & 9.30 & 57 & -25 & 44 & Right Postcentral Gyrus \\
\hline 3 & 6.50 & 45 & -25 & 17 & Right Heschls Gyrus
\end{tabular}

\section{Cluster 3 (357 vox)}

\begin{tabular}{llllll}
\hline 1 & 9.58 & -51 & -37 & 32 & Left SupraMarginal Gyrus \\
\hline 4 & 8.67 & -60 & -22 & 29 & Left Postcentral Gyrus \\
\hline
\end{tabular}

\section{Cluster 4 (22I vox)}

\begin{tabular}{lllllll}
\hline I & 8.4 & -39 & -4 & 5 & Left Insula Lobe \\
\hline 2 & 8.44 & -48 & -4 & 17 & Left Rolandic Operculum \\
\hline 3 & 7.15 & -51 & -1 & 32 & Left Precentral Gyrus \\
\hline 4 & 5.81 & -60 & 2 & 11 & Left Inferior Frontal Gyrus \\
\hline
\end{tabular}

Cluster 5 (208 vox)

\begin{tabular}{llllll}
\hline I & 9.27 & 24 & -40 & 65 & Right Postcentral Gyrus \\
\hline 2 & 9.26 & 30 & -46 & 56 & Right Inferior Parietal Lobule \\
\hline Cluster 6 (7 vox) & & & & & \\
\hline I & 6.40 & 45 & 11 & 5 & Right Insula Lobe \\
\hline
\end{tabular}

A summary of activation sites from SPM group analysis.

recorded inside the scanner were consistent with those recorded in two baseline (control) sessions (Figure 3, Table 1), suggesting there was no signal degradation or reduction of signal to noise ratio caused by conditions in the MRI environment or additional processing requirements. This is in agreement with a similar study using laser-evoked pain conducted by Iannetti and colleagues in 2005, which also showed no variation in amplitude or latency of $A \delta$ evoked potentials inside and outside the scanner. A study of auditory evoked potentials with concurrent fMRI has shown a difference in the latency and amplitude of the $\mathrm{N} 1$ component of the event related 


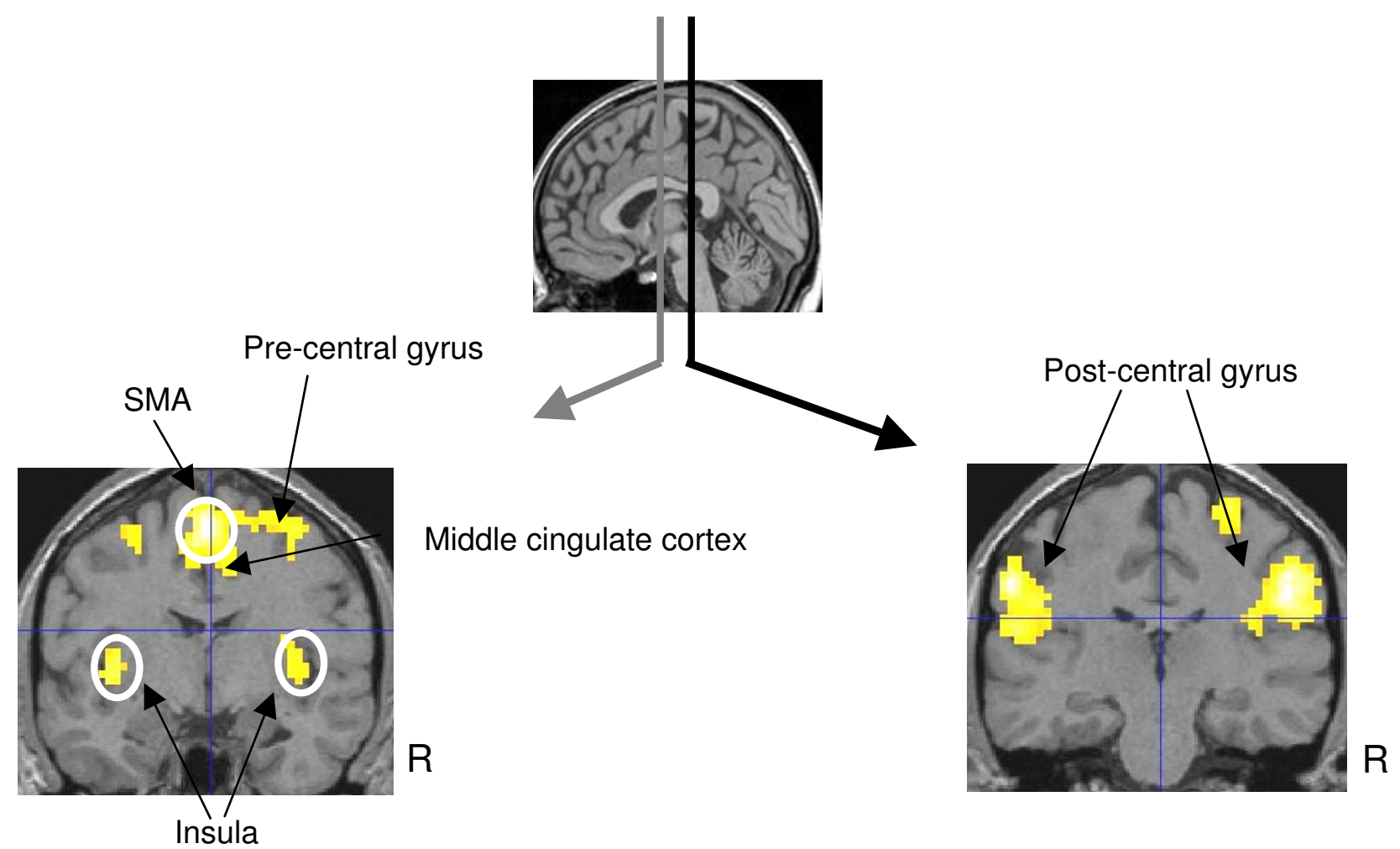

\section{Figure 4}

BOLD fMRI activation during simultaneous EEG. Noxious heat stimuli at $5 I^{\circ} \mathrm{C}$ caused BOLD fMRI responses bilaterally in the insula, post-central gyrus, and SMA and contralateral to the site of stimulation in the middle cingulate cortex and precentral gyrus. Random effect group analysis results $(p<0.05$ corrected) are displayed on structural images in neurological convention.

potential, with longer latencies and higher amplitudes recorded outside the scanner, however no difference in latency or amplitude of the P300 component of the response [5].

To avoid MRI induced artefacts contaminating EEG data, some groups have recorded EEG and fMRI separately [23], or employed an "interleaved" approach, where EEG is recorded during gaps in fMRI image acquisition to avoid artefacts in the EEG data, or short "burst" or "sparse" fMRI protocols are used, avoiding EEG data collection during image acquisition $[11,20,24-28]$. Despite avoiding most of the technical issues of using truly simultaneous EEG and AMRI, these interleaved protocols have theoretical drawbacks - the two methods are not monitoring the same neurophysiological event when they are recorded separately from one another, which is a disadvantage when the brain activity being studied is in any way unpredictable (such as spike activity in epilepsy, or sleep studies) or changeable (effects of habituation or learning).
Our contact heat evoked potential data has also highlighted a possible gender related difference in the latencies and amplitudes of responses - with female volunteers exhibiting an earlier latency and larger amplitude of response than male volunteers, and also a higher reported VAS score after CHEPS stimulation, an effect that has previously been noted [29]. However, in contrast to this study (which was also conducted in volunteers), there is no effect of subject age upon the latency and amplitude of evoked responses in our data set, or the VAS reported after contact heat stimulation. This may be due to the limited age range in our study, or the small number of subjects recruited. If the number of subjects was increased and the age range broadened, then an age dependent effect may be observed.

Prior to scanning volunteers we used phantom QA tests to assess whether the EEG recording equipment and the CHEPS stimulation device affected the quality of the fMRI images. This included signal-to-noise, ghosting measure- 


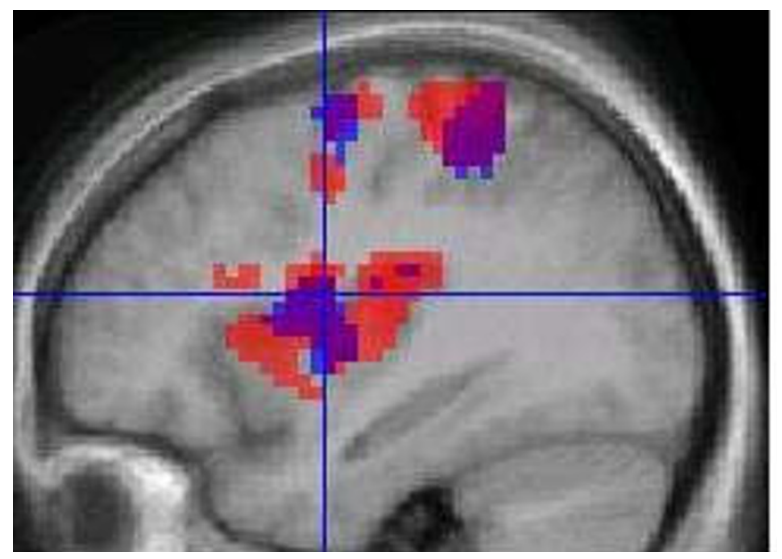

Figure 5

Reproducibility of $\mathrm{fMRI}$ activation over two sessions. Group results from each fMRI session are superimposed on the average high resolution structural scan. Session $I$ is shown in RED, session 2 in BLUE and areas that overlap in PURPLE. There is an overlap in the insula, post-central and pre-central gyrus.

ments and visual inspection of any artefacts. Our results showed that the fMRI images acquired alongside evoked potential recordings were unaffected by the simultaneous EEG acquisition, and were not impaired by the presence of the EEG recording and CHEPS equipment inside the scanner.

The results we obtained agree with previously published data showing patterns of brain activation after nociceptive and noxious thermal stimulation [10,11,18,30-34], with BOLD changes apparent in the insula, post-central gyrus, SMA, middle cingulate cortex and pre-central gyrus (Figure 4). Cooling of the CHEPS thermode back to baseline temperature will activate cool sensitive-fibres and this could potentially affect the BOLD response; however, the fMRI changes we observed were similar to those obtained with other methods of stimulation with noxious stimuli (these would be primarily related to the initial upstroke of the heat stimulus).

In accord with other studies of pain-evoked potentials, our data has revealed a significant positive correlation between evoked potential amplitude and reported pain scores or pain intensity $[15,29,32,35,36]$ (Figure 6a, b). However, this simple positive relationship with subjective pain scores was not present in the fMRI signal change data, even when regions of interest were analysed separately. This is unlike a recent study of painful electrical stimulation, which showed a positive correlation between stimulus correlated BOLD responses in a network of cor-
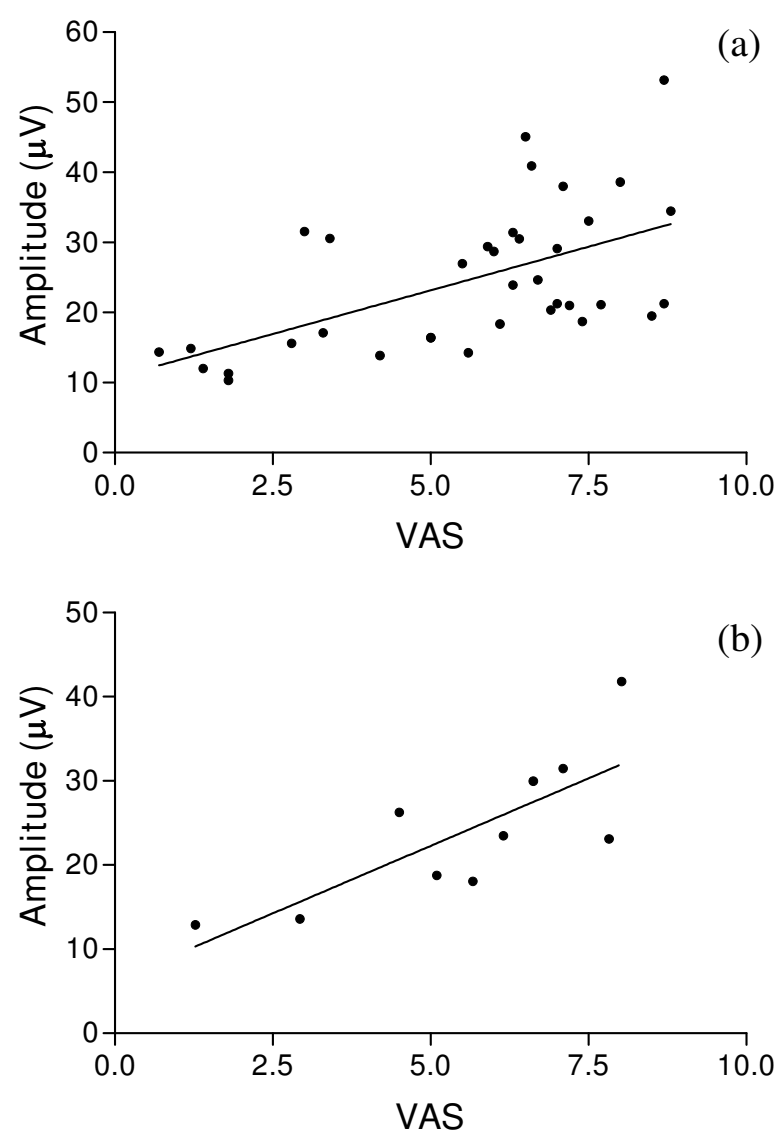

Figure 6

Correlation between evoked potential amplitude and pain score. (a) Correlation of amplitude and pain score data for all baseline and $f M R I$ sessions $(n=40)$ revealed a significant positive relationship $\left(r_{s}=0.5956, p<0.0001\right)$. (b) A significant, positive correlation $\left(r_{s}=0.7697, p=0.0063\right)$ was also seen when the baseline and $\mathrm{fMRI}$ data was pooled to give an overall evoked potential amplitude and VAS score for each subject $(n=10)$.

tical and cerebellar areas and reported pain intensity [11], and other studies using fMRI that have shown signal changes to correlate with pain intensity, or subjective experience of pain $[37,38]$. However these studies have either grouped subjects according to their sensitivity to heat stimuli [38], set stimuli according to individual thresholds [11], or used various stimulus intensities [37]. Our fMRI protocol used a stimulus at only one intensity for all subjects in the study, which may explain why no correlation with pain scores could be seen in our fMRI data. In protocols that lead on from this feasibility study stimuli at or just above the pain threshold for each individual will be used. While correlations were made with 
VAS scores as they are widely used in clinical trials of analgesics, these must be regarded with caution, as VAS scores are ordinal and not linear measures of pain perception further studies and analyses using an interval scale (Rasch model) would be of interest [39]).

\section{Conclusion}

This study demonstrates the feasibility of recording contact heat evoked potentials with simultaneous EEG and fMRI. Evoked potentials monitored inside the MRI scanner were similar to those recorded under baseline conditions and were highly reproducible on two occasions. We have also demonstrated a linear relationship between evoked potential amplitude and VAS score, as shown in previous volunteer studies. fMRI group analysis showed BOLD activation in areas shown to be associated with nociception in previous publications, but there was no simple correlation of regional fMRI signal changes with individual pain scores - suggesting changes in BOLD signal may reflect later processing in cerebral pathways rather than encoding pain intensity in a linear fashion. Further studies are in progress to demonstrate that simultaneous CHEPS-evoked potentials and fMRI is a useful tool in clinical trials of novel analgesics.

\section{Competing interests}

The authors declare that they have no competing interests.

\section{Authors' contributions}

KR was involved in setting up and carrying out the study, data analysis and writing the manuscript. AP was involved in setting up and carrying out the study, data analysis and writing the manuscript. CG was involved in carrying out the study and data analysis. MT was involved in setting up and carrying out the study and data analysis. DA was involved in setting up the study. RS was involved in carrying out the study. DM helped conceive the study and participated in its design and co-ordination, interpretation, and completion of the manuscript. PA conceived the study and participated in its design and co-ordination, interpretation, and completion of the manuscript. All authors read and approved the manuscript.

\section{Acknowledgements}

The authors would like to thank the BBSRC and also GlaxoSmithKline PLC for financial assistance, and in particular Dr Boris Chizh, Dr Zahid Ali, and Dr David Stephens of GlaxoSmithKline for helpful discussions.

\section{References}

I. Stern JM: Simultaneous electroencephalography and functional magnetic resonance imaging applied to epilepsy. Epilepsy Behav 2006, 8(4):683-692.

2. Czisch M, Wetter TC, Kaufmann C, Pollmacher T, Holsboer F, Auer DP: Altered processing of acoustic stimuli during sleep: reduced auditory activation and visual deactivation detected by a combined fMRI/EEG study. Neuroimage 2002, l 6(1):25I-258.
3. Goncalves SI, de Munck JC, Pouwels PJ, Schoonhoven R, Kuijer JP, Maurits NM, Hoogduin JM, Van Someren EJ, Heethaar RM, Lopes da Silva FH: Correlating the alpha rhythm to BOLD using simultaneous EEG/fMRI: inter-subject variability. Neuroimage 2006, 30(I):203-213.

4. Laufs H, Kleinschmidt A, Beyerle A, Eger E, Salek-Haddadi A, Preibisch C, Krakow K: EEG-correlated fMRI of human alpha activity. Neuroimage 2003, 19(4): I463-1476.

5. Mulert C, Jager L, Schmitt R, Bussfeld P, Pogarell O, Moller HJ, Juckel $G$, Hegerl U: Integration of fMRI and simultaneous EEG: towards a comprehensive understanding of localization and time-course of brain activity in target detection. Neuroimage 2004, 22(I):83-94.

6. Scarff CJ, Reynolds A, Goodyear BG, Ponton CW, Dort JC, Eggermont Jj: Simultaneous 3-T fMRI and high-density recording of human auditory evoked potentials. Neuroimage 2004, 23(3): I | 29-1 | 42 .

7. Comi E, Annovazzi P, Silva AM, Cursi M, Blasi V, Cadioli M, Inuggi A, Falini $A$, Comi $G$, Leocani $L$ : Visual evoked potentials may be recorded simultaneously with fMRI scanning: A validation study. Hum Brain Mapp 2005, 24(4):29I-298.

8. Henning S, Merboldt KD, Frahm J: Simultaneous recordings of visual evoked potentials and BOLD MRI activations in response to visual motion processing. NMR Biomed 2005, 1 8(8):543-552.

9. Lazeyras F, Zimine I, Blanke O, Perrig SH, Seeck M: Functional MRI with simultaneous EEG recording: feasibility and application to motor and visual activation. J Magn Reson Imaging 200I, 13(6):943-948.

10. lannetti GD, Niazy RK, Wise RG, Jezzard P, Brooks JC, Zambreanu L, Vennart W, Matthews PM, Tracey I: Simultaneous recording of laser-evoked brain potentials and continuous, high-field functional magnetic resonance imaging in humans. Neuroimage 2005, 28(3):708-719.

II. Christmann C, Koeppe C, Braus DF, Ruf M, Flor H: A simultaneous EEG-fMRI study of painful electric stimulation. Neuroimage 2007, 34(4): : 428-1437.

12. Lemieux L, Allen PJ, Franconi F, Symms MR, Fish DR: Recording of EEG during fMRI experiments: patient safety. Magn Reson Med 1997, 38(6):943-952.

13. Lemieux L, Allen P, Krakow K, Symms MR, Fish DR: Methodological Issues in EEG-correlated Functional MRI Experiments. IJBEM 1999, I (I):87-95.

14. Granovsky Y, Matre D, Sokolik A, Lorenz J, Casey KL: Thermoreceptive innervation of human glabrous and hairy skin: a contact heat evoked potential analysis. Pain 2005, I I 5(3):238-247.

15. Chen AC, Niddam DM, Arendt-Nielsen L: Contact heat evoked potentials as a valid means to study nociceptive pathways in human subjects. Neurosci Lett 200I, 3 I6(2):79-82.

16. Le Pera D, Valeriani M, Niddam D, Chen AC, Arendt-Nielsen L: Contact heat evoked potentials to painful and non-painful stimuli: effect of attention towards stimulus properties. Brain Topogr 2002, I 5(2): II5-123.

17. lannetti GD, Zambreanu L, Tracey I: Similar nociceptive afferents mediate psychophysical and electrophysiological responses to heat stimulation of glabrous and hairy skin in humans. J Physiol 2006, 577(Pt I):235-248.

18. Howard M, Coen S, Buchanan T, Smart T, Gregory S, Williams S, Huggins J, Hanna M: Test-retest Reproducibility of Cerebral and Subjective Responses to Painful and Non-painful Contact-Heat Evoked Potential Stimulation (CHEPS) [abstract]. Eur] Pain 2006, I O(SI):S82.

19. Greffrath W, Baumgartner U, Treede RD: Peripheral and central components of habituation of heat pain perception and evoked potentials in humans. Pain 2007.

20. Portas CM, Krakow K, Allen P, Josephs O, Armony JL, Frith CD: Auditory processing across the sleep-wake cycle: simultaneous EEG and fMRI monitoring in humans. Neuron 2000, 28(3):991-999.

21. Talairach J, Tournoux P: Co-planar stereotaxic atlas of the human brain. Thieme Publishing Group, New York; 1988.

22. Truini A, Galeotti F, Romaniello A, Virtuoso M, lannetti GD, Cruccu G: Laser-evoked potentials: normative values. Clin Neurophysiol 2005, II 6(4):82I-826.

23. Ball T, Schreiber A, Feige B, Wagner M, Lucking CH, Kristeva-Feige $\mathrm{R}$ : The role of higher-order motor areas in voluntary move- 
ment as revealed by high-resolution EEG and fMRI. Neuroimage 1999, I0(6):682-694.

24. Bonmassar G, Schwartz DP, Liu AK, Kwong KK, Dale AM, Belliveau JW: Spatiotemporal brain imaging of visual-evoked activity using interleaved EEG and fMRI recordings. Neuroimage 200I, I 3(6 Pt I): I035-1043.

25. Garreffa G, Bianciardi M, Hagberg GE, Macaluso E, Marciani MG, Maraviglia B, Abbafati M, Carni M, Bruni I, Bianchi L: Simultaneous EEG-fMRI acquisition: how far is it from being a standardized technique? Magn Reson Imaging 2004, 22(I 0): | 445-I 455.

26. Kruggel F, Herrmann CS, Wiggins CJ, von Cramon DY: Hemodynamic and electroencephalographic responses to illusory figures: recording of the evoked potentials during functional MRI. Neuroimage 200I, I4(6): I327-I336.

27. Muller BW, Stude P, Nebel K, Wiese H, Ladd ME, Forsting M, Jueptner M: Sparse imaging of the auditory oddball task with functional MRI. Neuroreport 2003, I4(I2): |597-I60I.

28. Nebel K, Stude P, Wiese H, Muller B, de Greiff A, Forsting M, Diener $\mathrm{HC}$, Keidel M: Sparse imaging and continuous event-related fMRI in the visual domain: a systematic comparison. Hum Brain Mapp 2005, 24(2): I 30-I43.

29. Cheng SS, Liu JY, Hsui YR, Chang ST: Chemical polymorphism and antifungal activity of essential oils from leaves of different provenances of indigenous cinnamon (Cinnamomum osmophloeum). Bioresour Technol 2006, 97(2):306-312.

30. Peyron R, Laurent B, Garcia-Larrea L: Functional imaging of brain responses to pain. A review and meta-analysis (2000). Neurophysiol Clin 2000, 30(5):263-288.

31. Ingvar M: Pain and functional imaging. Philos Trans $R$ Soc Lond $B$ Biol Sci 1999, 354( I 387): I 347-I358.

32. Qiu $Y$, Noguchi $Y$, Honda $M$, Nakata $H$, Tamura $Y$, Tanaka S, Sadato N, Wang $X$, Inui K, Kakigi R: Brain Processing of the Signals Ascending Through Unmyelinated C Fibers in Humans: An Event-Related Functional Magnetic Resonance Imaging Study. Cereb Cortex 2006, I6(9):। 289-I295.

33. Brooks JC, Nurmikko TJ, Bimson WE, Singh KD, Roberts N: fMRI of thermal pain: effects of stimulus laterality and attention. Neuroimage 2002, I 5(2):293-30I.

34. Moulton EA, Keaser ML, Gullapalli RP, Greenspan JD: Regional intensive and temporal patterns of functional MRI activation distinguishing noxious and innocuous contact heat. I Neurophysiol 2005, 93(4):2183-2193.

35. Ohara S, Crone NE, Weiss N, Treede RD, Lenz FA: Amplitudes of laser evoked potential recorded from primary somatosensory, parasylvian and medial frontal cortex are graded with stimulus intensity. Pain 2004, I I 0(I-2):3 I8-328.

36. Granovsky Y, Granot M, Nir RR, Yarnitsky D: Objective Correlate of Subjective Pain Perception by Contact Heat-Evoked Potentials. J Pain 2007.

37. Bornhovd K, Quante M, Glauche V, Bromm B, Weiller C, Buchel C: Painful stimuli evoke different stimulus-response functions in the amygdala, prefrontal, insula and somatosensory cortex: a single-trial fMRI study. Brain 2002, I 25(Pt 6): | 326-| 336.

38. Coghill RC, McHaffie JG, Yen YF: Neural correlates of interindividual differences in the subjective experience of pain. Proc Natl Acad Sci USA 2003, I 00( I 4):8538-8542.

39. Decruynaere C, Thonnard JL, Plaghki L: Measure of experimental pain using Rasch analysis. Eur J Pain 2007, I I (4):469-474.

\section{Pre-publication history}

The pre-publication history for this paper can be accessed here:

http://www.biomedcentral.com/1471-2253/8/8/prepub

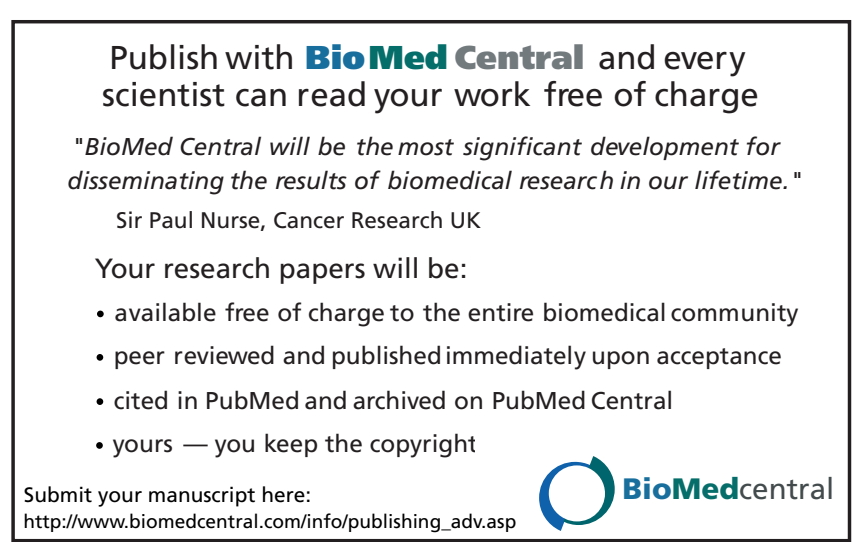

